\title{
Una visión general de la investigación sobre la enseñanza de las matemáticas en la educación de los estudiantes sordos
}

\author{
Sani de Carvalho Rutz da Silva ${ }^{1}$ \\ sani@utfpr.edu.br \\ https://orcid.org/0000-0002-1548-5739 \\ Elsa Midori Shimazaki \\ emshimazaki@uem.br \\ https://orcid.org/0000-0002-2225-5667 \\ Renata da Silva Dessbesel ${ }^{1}$ \\ renatadessbesel@utfpr.edu.br \\ https://orcid.org/0000-0002-2781-2397 \\ ${ }^{1}$ Universidade Tecnológica Federal do Paraná (UTFPR) \\ Ponta Grossa, Brasil \\ ${ }^{2}$ Universidade Estadual de Maringá (UEM) \\ Maringá, Brasil
}

Recibido: 10/04/2020 Aceptado: 19/05/2020

\section{Resumen}

El avance de la tecnología combinado con el desarrollo de nuevos recursos y la rapidez con que fluye la información, mantienen a la sociedad en un constante movimiento, y consecuentemente, la escuela como uno de los elementos de la sociedad sufre continuos cambios. Considerando este escenario, planteamos como objetivo del presente estudio diseñar un panorama de las investigaciones referentes la enseñanza de las matemáticas en la educación de personas sordas. Para este propósito utilizamos, como metodología de revisión la Methodi Ordinatio, para la selección y análisis de los artículos disponibles en la base de datos Scopus, Scielo, DOAJ y $E R I C$, a través de los descriptores: sordo, matemáticas, lenguaje de señas, escuela, tecnología y bilingüismo. Constatándose que la educación de los sordos se presenta en un ambiente de muchas posibilidades y, por lo tanto, diversos factores interfieren en el proceso de enseñanza y aprendizaje de las matemáticas de estas personas en el aula. Los resultados revelaron que entre los factores que influyen, los puntos convergentes son la enseñanza y el aprendizaje de las matemáticas en el lenguaje de señas, con el desarrollo de herramientas visuales y la construcción de un ambiente accesible para los sordos, con respeto y valorización a la cultura sorda.

Palabras clave: Lenguaje de señas. Enseñanza y aprendizaje. Sordez

\section{Um panorama das investigações sobre o ensino de matemática na educação de surdos}

\section{Resumo}

O avanço da tecnologia aliado ao desenvolvimento de novos recursos e a rapidez de informações mantêm a sociedade em constante movimento e, consequentemente, a escola como um dos segmentos sociais, passa por contínuas mudanças. Ao considerar tal fato, o objetivo deste estudo é desenhar um panorama das investigações sobre o ensino de matemática na educação de surdos. Para tanto, utilizou-se, como metodologia de revisão, a Methodi Ordinatio para seleção e análise dos artigos disponíveis nas bases de dados Scopus, Scielo, DOAJ e ERIC, por meio dos 
descritores: surdos, matemática, língua de sinais, escola, tecnologia e bilinguismo. Constatouse que a educação de surdos se apresenta em um ambiente de muitas possibilidades e por conseguinte, diversos fatores interferem no processo de ensino e aprendizagem de matemática em sala de aula a essas pessoas. Os resultados revelaram que dentre os fatores que influenciam, os pontos convergentes são, o ensino e a aprendizagem de matemática em língua de sinais, com desenvolvimento de ferramentas visuais e a construção de um ambiente acessível para os surdos, com respeito e valorização à cultura surda.

Palavras chave: Língua de sinais. Ensino e aprendizagem. Surdez.

\title{
An overview of research on the teaching of mathematics in deaf students' education
}

\begin{abstract}
The technology advances combined with the development of new resources and the speed of information keep the society in constant movement and, therefore, the school as a social segment undergoes continuous changes. Considering this fact, this study aims to draw a research panorama about mathematics teaching in deaf students' education. The Methodi Ordinatio was used as review methodology to select and analyze the articles available on Scopus, Scielo, DOAJ and ERIC databases, throughout the descriptors: deaf, mathematics, sign language, school, technology and bilingualism. The deaf people's education, was concluded, is presented in an environment with many possibilities and thus various factors which interfere in mathematics teaching-learning process in classroom of these students. The results showed that among the factors which influence, the convergent aspects are the mathematics teaching-learning in sign language, development of visual tools and the constructions of an accessible environment to deaf people and respect and appreciation for deaf culture.
\end{abstract}

Keywords: Sign language. Teaching-learning. Deafness.

\section{Considerações iniciais}

A matemática contempla diversos contextos de aplicação nos campos das ciências e das engenharias, e, como aliado, os avanços tecnológicos permitem a exploração destes contextos de modo dinâmico e eficiente. De acordo com D’Ambrosio (2018) novos estilos de matemática são emergentes, de modo que a tecnologia possa auxiliar no processo de ensino e aprendizagem de conceitos científicos e promover uma educação voltada às práticas sociais.

A pesquisa em sala de aula constitui um meio de investigar as possibilidades e as fragilidades para a aprendizagem de matemática, como afirmam Borba, Almeida e Gracias (2018), a pesquisa é um dos elementos que possibilita as mudanças no ambiente escolar. Dessa forma, a investigação surge da necessidade, dos desafios e dos obstáculos vivenciados por professores e pesquisadores, cujo objetivo é provocar transformações nas metodologias e nas práticas da escola (Borba et al., 2018).

O processo de ensino e aprendizagem de matemática requer atenção da comunidade acadêmica no que se refere à inclusão, especialmente a metodologias de ensino que se possam 
ter acesso ao currículo e estrutura didático-pedagógica para atender a diversidade, de forma que todos se apropriem do conhecimento escolar necessários ao desenvolvimento do homem e, por sua vez, da cidadania. No caso desta pesquisa, especificamente, refere-se às pessoas surdas, que compartilham da comunidade surda por suas características culturais, no entanto, na análise dos resultados são considerados os termos originais dos autores surdos e deficientes auditivos. Muitos avanços foram conquistados na educação de surdos, Scheetz (2012) explica que, ao longo da história, os surdos vivenciaram muitas mudanças devido à redução de barreiras globais, ao avanço tecnológico e ao acesso aos dados de pesquisas em uma esfera mundial.

As pesquisas proporcionam discussões em torno da educação de surdos, como as barreiras de comunicação e a influência da língua de sinais no processo de ensino e aprendizagem, temas centrais, que necessitam da compreensão dos educadores. A comunicação possibilita a expressão da história, das imagens e da interpretação do mundo, assim e, "A língua de sinais é para os surdos uma adaptação única a um outro modo sensorial; mas é. também, e igualmente, uma corporificação da identidade pessoal e cultural dessas pessoas” (Sacks, 2010, p. 105).

Os estudantes surdos, assim como os ouvintes, carregam uma bagagem cultural e linguística que requer respeito e conhecimento dos professores ao planejar suas práticas, de modo que é necessário sua implementação no currículo (Scheetz, 2012). Ao encontro dessa ideia, Soares e Sales (2018) afirmam que a aprendizagem de matemática na educação de surdos é beneficiada com a incorporação dos recursos visuais. Os autores alertam que a falta da relação entre o conteúdo de matemática e a vida cotidiana, provoca um distanciamento entre a escola, a matemática e a realidade dos alunos.

Diante desse cenário, apresenta-se, neste texto, um panorama das investigações direcionadas ao ensino de matemática na educação de surdos. O estudo partiu das seguintes questões de pesquisa:

- Quais temas são investigados e publicados sobre a interface educação de surdos e ensino de matemática?

- Quais fatores influenciam o processo de ensino e aprendizagem de matemática para estudantes surdos? 
Espera-se com este estudo contribuir para que professores e pesquisadores interessados nesta abordagem ampliem seu conhecimento na interface educação de surdos e ensino de matemática.

\section{Aporte teórico}

O avanço da tecnologia aliado ao desenvolvimento de novos recursos e à rapidez de informações promove mudanças significativas no contexto escolar. A matemática, por sua vez, tem papel essencial nesse momento e, consequentemente, altera as competências necessárias para os alunos perante a sociedade (Gravemeijer; Stephan; Julie; Lin; Ohtani, 2017).

Ambientes tecnológicos para o ensino de matemática são uma das possibilidades na educação de surdos, pois, como destacado por Adamo-Villani e Wilbur (2010), foram desenvolvidos com um design visual atraente, com estratégias de games, a envolver os estudantes e estimular a participação. Como exemplo, os autores apresentam o SMILE, ambiente de aprendizagem virtual imersivo para crianças, projetado com o objetivo para disciplinas no campo da matemática, ciências e tecnologia, com a interação em Língua de Sinais Americana (ASL).

De acordo com Mendes, Bueno, Dessbesel e Silva (2019), a gamificação também pode ser uma estratégia viável na educação de surdos, ao potencializar aspectos como motivação e interação nas aulas, como, também, trazer a possibilidade do trabalho inclusivo e a aprendizagem da Língua Brasileira de Sinais (Libras). Outras possibilidades, como o uso de materiais concretos e visuais, foram apontados em Dessbesel, Silva e Shimazaki (2018) como facilitadores, ao analisarem pesquisas sobre o ensino de matemática para estudantes surdos.

Da mesma forma, Sales (2009) observou que o uso de recursos tecnológicos favorece a comunicação, a atender as necessidades dos educandos, assim como o uso de estratégias visuais colabora com o trabalho do professor e auxilia no domínio dos conceitos formais de matemática. A autora destaca que, nesse trabalho, alguns aspectos necessários, tais como o uso de imagens, textos curtos e a Libras para tornar o ambiente virtual acessível aos surdos.

Karnopp, Pokorski e Zanini (2019, p. 3) consideram “[...] os surdos como sujeitos pertencentes a uma minoria linguística, vinculados a uma comunidade que compartilha códigos culturais". Nesse sentido ressalta-se a importância da comunicação em sala de aula a partir da 
primeira língua dos surdos, isto é, a língua de sinais e o conhecimento dos aspectos da cultura, que influenciam a aprendizagem.

A língua de sinais apresenta uma relação estreita com o processo de ensino e aprendizagem dos estudantes surdos. Nesse sentido, a pesquisa de Costa e Silveira (2019) mostra que a apresentação de conteúdo em Libras aliado aos aspectos visuais, auxilia na compreensão dos conceitos pelos estudantes surdos. Ao encontro Karnopp et al. (2019) explicam que a língua de sinais está no centro dos objetivos da comunidade surda e na luta por escolas bilíngues.

De acordo com Costa e Silveira (2019) no contexto das aulas inclusivas decorre que muitos docentes desconhecem a língua de sinais, desse modo, torna-se essencial que usem de estratégias e metodologias que valorizem o visual. Assim, os apoiam o processo de interpretação e tradução dos conceitos para Libras, pelos profissionais intérpretes que, na maioria dos casos, não possuem formação em Matemática. Para Segadas, Bernardo, Moreira, Barbosa, Santos e Garcez (2018) somente a presença do intérprete e a tradução para língua de sinais não são aspectos suficientes para a aprendizagem, de modo que o trabalho conjunto entre professor e intérprete no planejamento das atividades é fundamental.

No ensino de matemática de qualidade para os estudantes surdos, precisa-se considerar questões como acesso ao conteúdo em Libras, a atenção aos sinais utilizados, que, muitas vezes, não apresentam sinais para termos específicos dos conteúdos, além de estar apoiado na tríade língua de sinais, conhecimento matemática e metodologia apropriada (Arroio; Pereira; Pinto; Esquincalha, 2016).

O contato com a língua de sinais e a cultura surda é essencial na formação dos estudantes surdos, Holcomb (2011) explica que, em sua maioria, os surdos são filhos de pais ouvintes não fluentes em língua de sinais, o que dificulta o acesso às informações do mundo exterior. $\mathrm{O}$ autor traz uma crítica às escolas, ao afirmar que muitas das políticas educacionais são organizadas por pessoas com pouco conhecimento sobre a comunidade surda, como reflexo, a falta da língua de sinais ser amplamente adotada no ambiente escolar, a torná-la uma situação difícil para os surdos. Também complementa: "Em razão de ambientes linguísticos e comunicativos empobrecidos em casa e na escola, muitas crianças surdas crescem com habilidade de leitura e escrita relativamente deficientes" (Holcomb, 2011, p. 141). 
Na perspectiva da inclusão Arroio et al. (2016) explicam que os professores precisam se dedicar ao planejar suas atividades para a aula de Matemática, de modo a conhecer a cultura e as especificidades da comunicação para o sucesso do processo de ensino e aprendizagem de matemática, como, por exemplo, explorar as competências visual-espacial para a construção do conhecimento. Os autores destacam estratégias como o uso das tecnologias, por meio de vídeos e de softwares de matemática, a tornar as aulas mais dinâmicas e facilitando a comunicação entre professor e aluno.

Nesse sentido, considera-se que a escola tem um papel fundamental, a configur-se como um ambiente em que os surdos compartilham conhecimento de sua cultura, em muitas situações sendo o único lugar que isto acontece (Karnopp et al., 2019). Tal situação ocorre uma vez que as crianças surdas apresentam, de acordo com Segadas et al. (2018), graus diferentes de conhecimentos e fluências da língua de sinais, o que depende das experiências que vivenciaram em diversos contextos em que convivem.

\section{Metodologia}

Este estudo consiste em uma Revisão Sistemática da Literatura, a partir das orientações de Pagani, Kovaleski e Resende (2015) denominada Methodi Ordinatio, que compreende nove etapas para a constituição de um portfólio bibliográfico, a levar em consideração o ano de publicação, o número de citações e o fator de impacto dos artigos publicados em periódicos, de maneira a classificar os melhores estudos na temática que se pretende pesquisar. A seguir apresenta-se as nove etapas:

Etapa 1 - Estabelecimento da intenção de pesquisa: neste estudo, objetiva-se desenhar um panorama das investigações sobre o ensino de matemática na educação de surdos, de modo a estabelecer os principais autores, temas e considerações, a contribuir para o avanço das pesquisas no âmbito da educação de surdos.

Etapa 2 - Pesquisa preliminar: foram selecionadas e testadas combinações de palavras em diferentes bases, para se estabelecer a busca definitiva.

Etapa 3 - Definição das palavras - chave e bases de dados: como filtros nas bases de dados foram selecionados somente artigos em periódicos e a busca foi realizada nos campos títulos, resumo e palavras-chave. Palavras-chave: a) deaf* AND math* AND "sign language", b) deaf* AND math* AND school, c) deaf* AND math* AND technology d) deaf* AND math* AND 
biblingual*. Bases: Scientific Electronic Library Online (SciELO), Scopus, Education Resources Information Center (ERIC), Web of Science, Directory of Open Access Journals (DOAJ).

Etapa 4 - Pesquisa final nas bases: a pesquisa foi realizada em janeiro de 2020. Resultou em 429 artigos, sendo 22 SciELO; 185 Scopus; 39 ERIC; 112 Web of Science; 71 DOAJ.

Etapa 5 - Filtragem: foram aplicados procedimentos de filtragem dos dados a partir de critérios pré-estabelecidos, artigos duplicados, artigos com caráter de entrevistas, estudos clínicos, resumos não localizados e pesquisas não direcionadas ao ensino de matemática para estudantes surdos. Na Tabela 1 sintetizaram-se os resultados dos dados:

Tabela 1

Filtragem dos artigos

\begin{tabular}{lcc}
\multicolumn{1}{c}{ Pesquisas/critérios } & Quantidade & $\%$ \\
\hline Total de artigos & 429 & $100 \%$ \\
Eliminados por duplicação & 178 & $41,49 \%$ \\
Eliminados por entrevistas e estudos clínicos & 6 & $1,40 \%$ \\
Eliminados por resumos não localizados & 3 & $0,70 \%$ \\
Eliminados por não estar de acordo com o foco da investigação & 132 & $30,77 \%$ \\
Total de artigos descartados & 319 & $74,36 \%$ \\
Total de artigos do portifólio & 110 & $25,64 \%$
\end{tabular}

Fonte: Elaboração dos autores

Etapa 6 - Identificação do Fator de Impacto e do número de citações: após o portifólio constituído por 110 pesquisas, identificou-se o Journal Citation Reports (JCR), e ao não existir, empregou-se a métrica CiteScore, para os estudos selecionados. Para o número de citações foi utilizado o Google Acadêmico.

Etapa 7 - Ranking: aplicou-se a equação InOrdinatio, proposta por Pagani et al. (2015) como InOrdinatio $=(\mathrm{IF} / 1000)+\alpha *[10-($ ano da pesquisa - ano da publicação $)]+\left(\sum \mathrm{Ci}\right)$, em que $\alpha$ corresponde ao fator de ponderação de 1 à 10 e afeta o grau de importância do critério ano, IF é o fator de impacto do periódico e Ci número de citações do artigo. De acordo com Pagani et al. (2015) esta equação estabelece uma relação entre as métricas: número de citações, fator de impacto e ano de publicação; com o propósito de estabelecer um ranking entre os artigos que compõem o portifólio. Neste estudo, utilizou-se $\alpha=5$, por considerar a importância de pesquisas recentes, mas, também, incluir os estudos clássicos. Como explicado por Pagani, 
Kowaleski e Resende (2018), os estudos que têm um número elevado de citações colaboram no fator de impacto atual das revistas, mesmo que seja um artigo antigo.

Etapa 8 - Busca dos artigos completos: todos os artigos foram armazenados em sua versão completa.

Etapa 9 - Leitura e análise dos artigos: das pesquisas que constituem o portifólio, foram extraídos dados quantitativos que estabelecem a primeira parte da análise, isto é, a visão geral das publicações, com os 110 estudos localizados e escolhidos. Na segunda parte, foram considerados para a análise qualitativa os artigos com InOrdinatio no ranking > 47,4 (valor obtido a partir da média aritmética dos valores positivos, como ponto de corte), justifica-se esse corte por incluir os estudos com expressivo número de citações, como, também englobar os estudos mais recentes. Ao todo foram incluídos, na segunda parte, 24 estudos.

A seguir, discorrem-se sobre os resultados do estudo de revisão.

\section{Resultados e Discussões}

No século XX houve um crescimento no interesse em relação ao desempenho dos alunos surdos, o que possibilitou descobertas, especialmente em resultados de testes padronizados, a mostra que os estudantes tendem a ter resultado inferior comparado aos seus pares ouvintes (Moores, 2017). As pesquisas de Kritzer e Pagliaro (2013) mostraram um baixo desempenho dos alunos surdos no desenvolvimento de atividades que envolvem resolução de problemas. Do mesmo modo Barbosa (2014), ao trabalhar com crianças surdas observou um desempenho menor ao ser comparado com o de crianças ouvintes.

Os recursos tecnológicos, o uso de recursos materiais para sala de aula, as discussões sobre a fluência da língua de sinais e seus reflexos na aprendizagem, assim como a formação de professores são temas encontrados a partir da análise dos dados. Como ponto convergente, os estudos apresentam resultados sobre o ensino e aprendizagem de matemática na educação de surdos.

Dessa forma, observaram-se as características dos estudos sobre a educação de surdos, como os temas principais que foram abordados, número de publicações por revistas e principais autores, nos 110 artigos que compõem o portifólio deste estudo. No Gráfico 1, apresentam-se as publicações por temas, a partir de categorias pré-organizadas e observa-se que o primeiro estudo incluído foi publicado em 1974, por Culbertson com uma abordagem do uso da Instrução 
Assistida por Computador (CAI), com estudantes surdos em 15 escolas diferentes, durante 3 anos. De acordo com Culbertson (1974) foi observado, a partir da aplicação de testes, que o desempenho dos estudantes surdos em matemática era maior após o treinamento com CAI, a constituir-se em uma ferramenta efetiva para o ensino.

Gráfico 1: Número de publicações por temas

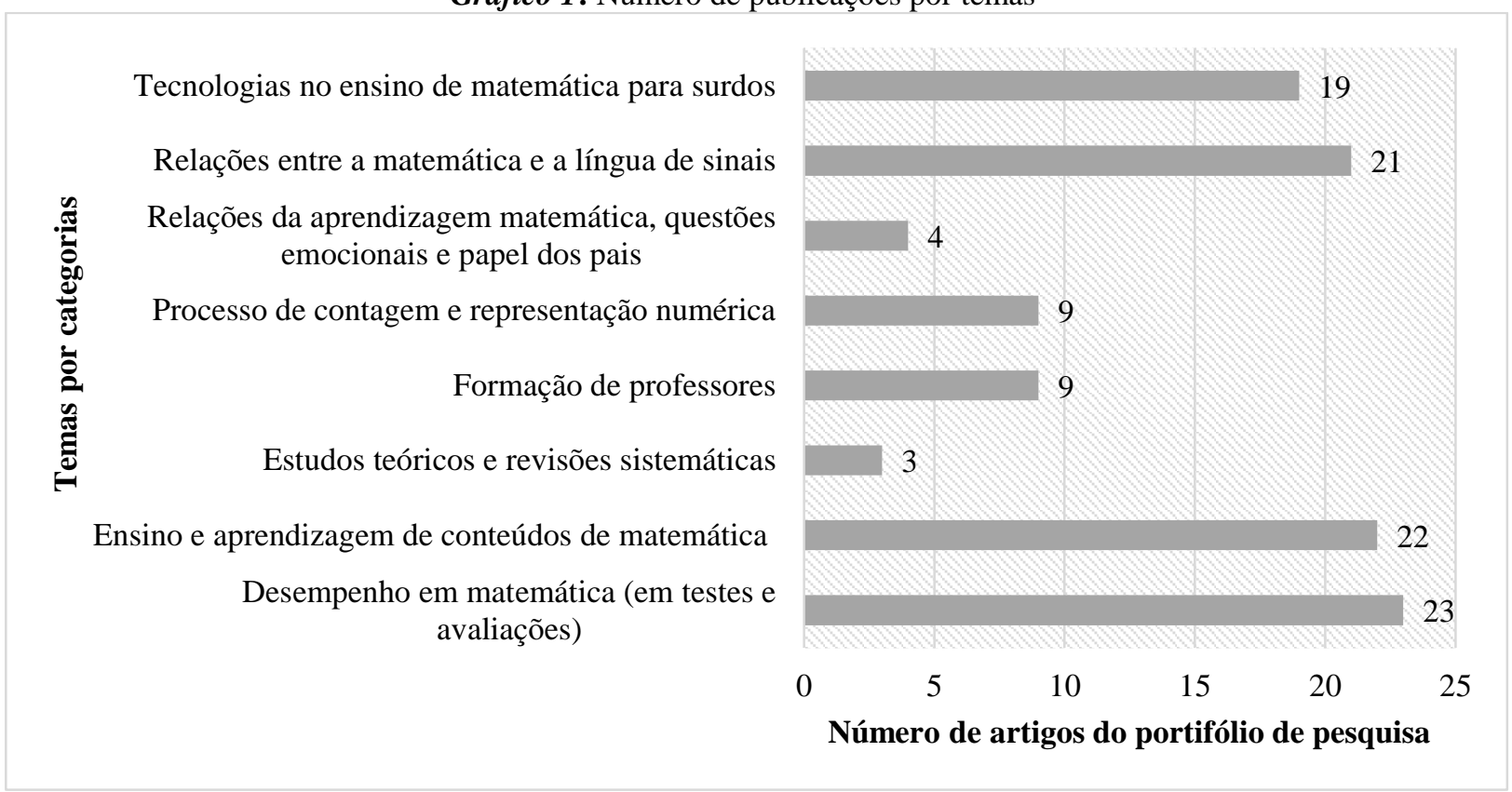

Fonte: Elaboração dos autores, 2020

O tema de investigação mais presente nas pesquisas é o desempenho em matemática, principalmente oriundos de pesquisadores dos Estados Unidos e com base em testes nacionais padronizados. As crianças surdas, podem apresentar, mesmo antes da etapa de escolarização formal, atrasos acadêmicos (Kritzer, 2009). Nessa mesma linha Marschark, Shaver, Nagle e Newman (2015) mostram que o desempenho dos estudantes surdos e deficientes auditivos nos testes, tem uma série de fatores envolvidos, como suas experiências escolares e ambiente familiar.

As tecnologias no ensino de matemática para surdos apresentam um valor significativo e crescente (de 5 para 10 pesquisas) nos últimos cinco anos, como mostra a Tabela 2. Esses estudos apresentam entre outros temas, o uso de avatares para auxiliar o processo de ensino e aprendizagem e facilitar a comunicação em sala de aula. Os avatares fazem uso de um personagem animado 3D em língua de sinais, podendo ser usado por estudantes e seus pais ouvintes, a tornar acessível os conteúdos digitais nessa língua, como também ampliar a interação com os materiais de aprendizagem (Adamo-Villani; Hayward, 2011). 
Tabela 2

Número de publicações por temas e ano

\begin{tabular}{|c|c|c|c|c|c|c|c|c|}
\hline $\begin{array}{c}\text { Temas x Número de } \\
\text { publicações no período de } 5 \\
\text { anos }\end{array}$ & $\begin{array}{c}1974 \text { a } \\
1979\end{array}$ & $\begin{array}{c}1980 \text { a } \\
1985\end{array}$ & $\begin{array}{c}1986 \text { a } \\
1991\end{array}$ & $\begin{array}{c}1992 \text { a } \\
1997\end{array}$ & $\begin{array}{c}1998 \text { a } \\
2003\end{array}$ & $\begin{array}{c}2004 \text { a } \\
2009\end{array}$ & $\begin{array}{c}2010 \mathrm{a} \\
2015\end{array}$ & $\begin{array}{c}2016 \text { a } \\
2020\end{array}$ \\
\hline $\begin{array}{l}\text { Desempenho em matemática (em } \\
\text { testes e avaliações) }\end{array}$ & & 2 & 1 & 2 & 1 & 6 & 6 & 5 \\
\hline $\begin{array}{l}\text { Ensino e aprendizagem de } \\
\text { conteúdos de matemática }\end{array}$ & 1 & 1 & & & 2 & 5 & 5 & 8 \\
\hline $\begin{array}{l}\text { Estudos teóricos e revisões } \\
\text { sistemáticas }\end{array}$ & 1 & & & & & 1 & & 1 \\
\hline Formação de professores & & & & & 2 & 1 & 4 & 2 \\
\hline $\begin{array}{l}\text { Processo de contagem e } \\
\text { representação numérica }\end{array}$ & & & & & 1 & 3 & 5 & \\
\hline $\begin{array}{l}\text { Relações da aprendizagem } \\
\text { matemática, questões emocionais } \\
\text { e papel dos pais }\end{array}$ & & & 1 & & & 1 & & 2 \\
\hline $\begin{array}{l}\text { Relações entre a matemática e a } \\
\text { língua de sinais }\end{array}$ & & & & 1 & 1 & 3 & 9 & 7 \\
\hline $\begin{array}{l}\text { Tecnologias no ensino de } \\
\text { matemática para surdos }\end{array}$ & 1 & 1 & & & & 2 & 5 & 10 \\
\hline Total & 3 & 4 & 2 & 3 & 7 & 22 & 34 & 35 \\
\hline
\end{tabular}

Fonte: Elaboração dos autores

As mudanças são necessárias para o avanço no campo de pesquisa, assim entre os temas, verifica-se que, atualmente, há uma ênfase com relação ao processo de ensino e aprendizagem de matemática a partir da abordagem de diversos conteúdos de álgebra, geometria, número e operações e sistemas de medidas, como, também, o uso de tecnologias e as relações entre a matemática e a língua de sinais.

As pesquisas mostram alternativas para a educação de surdos, como as experiências de Jannahe e Prahmana (2019), ao relatar sobre o uso de um contexto aplicado, com situações reais a favorecer a compreensão do conceito de fração e apresentar melhoria no desempenho dos estudantes surdos. Outro exemplo é a falta de sinais específico em Libras para a matemática, apontado como obstáculo com atividades de geometria, no entanto, a negociação de sinais em sala de aula torna-se uma importante ferramenta (Sales; Penteado; Moura, 2015).

No Gráfico 2 os estudos foram divididos por área de investigação de acordo com a classificação da Revista na plataforma da Scopus ( a considerar que a mesma revista pode estar em mais de uma área), para as revistas que não possuem tal classificação, foi realizada pelos autores de acordo com o escopo publicado na página da revista. 
Gráfico: 2 Área de publicação das revistas

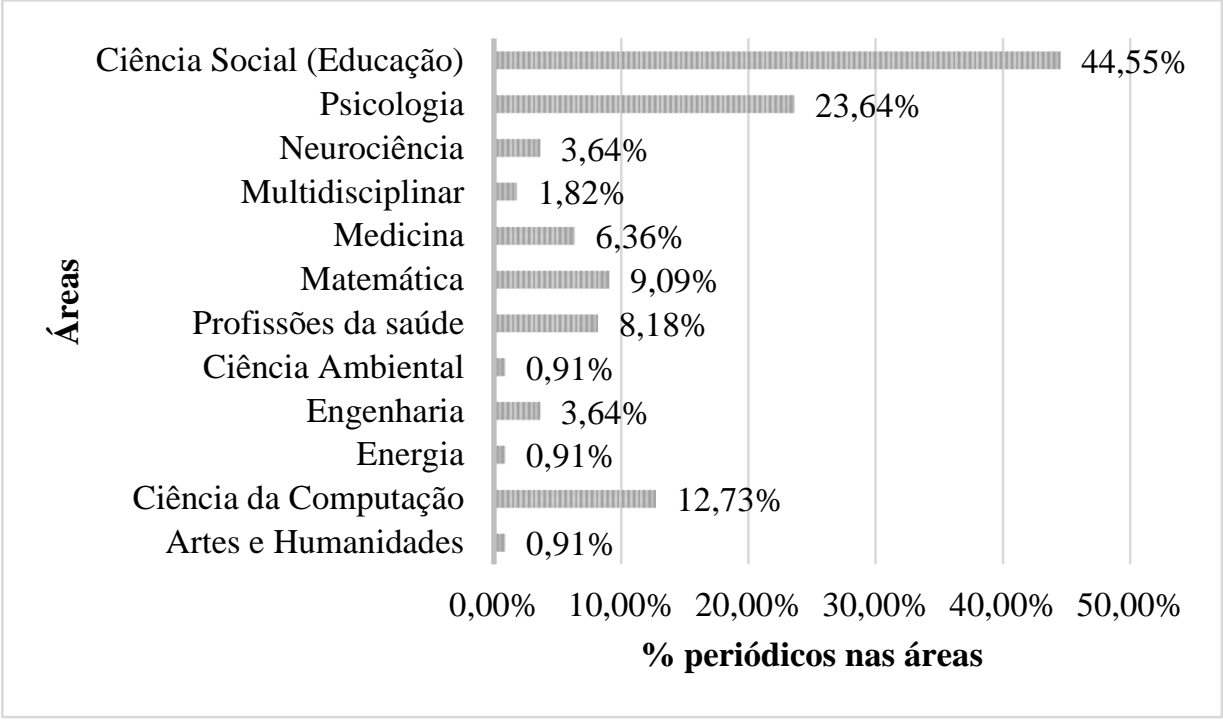

Fonte: Elaboração dos autores, 2020

Observa-se no Gráfico 2 que a maior parte das pesquisas foi publicada em periódicos classificados na área de Educação (38\%) e Psicologia (20\%), nesta última, em especial as pesquisas sobre a influência da língua de sinais. Ao mesmo tempo, a outra parcela é dividida em diversas áreas, com destaque para a Ciência da Computação, com contribuições tecnológicas, como o uso de avatares que fazem a tradução para a língua de sinais.

A Tabela 3 apresenta as cinco revistas selecionadas que mais publicaram sobre o tema a compor esta pesquisa em relação ao número de publicações por ano, considerando o foco central sobre educação de surdos e ensino de matemática.

\section{Tabela 3}

Número de publicações por revista por ano

\begin{tabular}{|c|c|c|c|c|c|c|c|c|}
\hline Revistas & $\begin{array}{c}1974 \text { a } \\
1979\end{array}$ & $\begin{array}{c}1980 \text { a } \\
1985\end{array}$ & $\begin{array}{c}1986 \text { a } \\
1991\end{array}$ & $\begin{array}{c}1992 \text { a } \\
1997\end{array}$ & $\begin{array}{c}1998 \text { a } \\
2003\end{array}$ & $\begin{array}{c}2004 \text { a } \\
2009\end{array}$ & $\begin{array}{c}2010 \mathrm{a} \\
2015\end{array}$ & $\begin{array}{c}2016 \text { a } \\
2020\end{array}$ \\
\hline American Annals of the Deaf & 3 & 2 & & 2 & 4 & 7 & 1 & 1 \\
\hline Caderno CEDES & & & & & & & 3 & \\
\hline Cognition & & & & & & & 3 & \\
\hline Exceptional Children & & 1 & 1 & & & & 1 & \\
\hline $\begin{array}{l}\text { Journal of Deaf Studies and Deaf } \\
\text { Education }\end{array}$ & & & & & & 10 & 5 & 3 \\
\hline
\end{tabular}

Fonte: Elaboração dos autores

As duas revistas com maior número de publicações na interface educação de surdos e ensino de matemática são Amercian Annals of the Deaf and Journal e Deaf Studies and Deaf Education, ambas com espoco direcionado à temática da educação de surdos e possuir fator de impacto, Journal Citation Reports (JCR) 0,638 e 1,556 respectivamente, o que mostra que as 
pesquisas publicadas envolvem estudos relevantes no campo. Esta informação torna-se importante para que professores e pesquisadores envolvidos com o tema encontrem resultados de estudos ao longo dos anos e que fundamentem as suas pesquisas.

Com relação aos autores principais, na Tabela 4, apresenta-se a relação de autoria e coautoria, a considerar os primeiros autores das publicações com dois ou mais artigos no portifólio. Esses dados contribuem aos pesquisadores da área para o acesso as pesquisas em educação de surdos e ensino de matemática.

Tabela 4

Número de publicações como autor principal e em coautorias no portifólio

\begin{tabular}{|c|c|c|c|c|}
\hline Nome & Filiação & País & $\begin{array}{c}\text { Autor } \\
\text { Principal }\end{array}$ & Coautorias \\
\hline $\begin{array}{l}\text { Carrie Lou } \\
\text { Garberoglio }\end{array}$ & Universidade do Texas em Austin & Estados Unidos & 1 & 1 \\
\hline Claudia M. Pagliaro & Universidade do Estado de Michigan & Estados Unidos & 5 & 3 \\
\hline Elizabet Spaepen & Universidade de Chicago & Estados Unidos & 1 & 1 \\
\hline Ellen Ansell & Universidade de Pittsburgh & Estados Unidos & 1 & 1 \\
\hline Evelyn Silvia & Universidade da Califórnia & Estados Unidos & 1 & 1 \\
\hline Gary Blatto-Vallee & Universidade de Aberdeen & Escócia & 1 & 2 \\
\hline Georgianna Borgna & Instituto Técnico Nacional para Surdos & Estados Unidos & 1 & 1 \\
\hline Heloiza H. Barbosa & Universidade Federal de Santa Catarina & Brasil & 2 & 0 \\
\hline Judy Vesel & TERC & Estados Unidos & 2 & 0 \\
\hline Karen Kritzer & Universidade do Estado de Kent & Estados Unidos & 2 & 2 \\
\hline Katherine Nagle & SRI Internacional & Estados Unidos & 1 & 1 \\
\hline Lynn A. Newman & SRI Internacional & Estados Unidos & 1 & 2 \\
\hline Marc Marschark & Instituto Técnico Nacional para Surdos & Estados Unidos & 3 & 3 \\
\hline Marrie Coppola & Universidade de Connecticut & Estados Unidos & 1 & 1 \\
\hline $\begin{array}{l}\text { Nicoletta Adamo- } \\
\text { Villani }\end{array}$ & Purdue University & Estados Unidos & 4 & 0 \\
\hline $\begin{array}{l}\text { Nohemy Marcela } \\
\text { Bedoya Rios }\end{array}$ & $\begin{array}{l}\text { Universidade Federal do Rio Grande do } \\
\text { Sul }\end{array}$ & Brasil & 2 & 0 \\
\hline Rebecca Bull & Universidade de Aberdeen & Escócia & 2 & 1 \\
\hline Ronald R. Kelly & Instituto Técnico Nacional para Surdos & Estados Unidos & 1 & 2 \\
\hline Stephanie W. Cawthon & Universidade do Texas em Austin & Estados Unidos & 4 & 2 \\
\hline Suriza van der Sandt & Faculdade de New Jersey & Estados Unidos & 1 & 1 \\
\hline Susan Goldin-Meadow & Universidade de Chicago & Estados Unidos & 1 & 2 \\
\hline Susan R. Easterbrooks & Universidade do Estado de Georgia & Estados Unidos & 1 & 1 \\
\hline Terezinha Nunes & Universidade de Londres & Londres & 2 & 1 \\
\hline
\end{tabular}

Fonte: Elaboração dos autores

Os pesquisadores dos Estados Unidos aparecem em maior número, como também há muitas colaborações entre si nas diversas pesquisas da Universidade do Texas, da Universidade 
Michigan, da Universidade de Gallaudet e do National Technical Institute for the Deaf, entre outros. No Brasil pesquisas de Barbosa $(2013,2014)$ trazem importantes contribuições sobre o desempenho em testes e atividades de sala de aula na educação de surdos, como também a pesquisa de Ríos e colaboradores (Ríos; Guimarães; Dorneles, 2018; Ríos; López; Gallo, 2013). Como complemento, no cenário brasileiro de pesquisa, outras pesquisas também compõem o portifólio deste estudo, entre elas Fernandes e Healy (2013), Peixoto (2015), Sales et. al (2015), Madalena, Correa e Spinillo (2020) de modo que trazem contribuições sobre práticas em sala de aula com estudantes surdos.

Com o objetivo de responder à segunda questão de pesquisa, com relação aos fatores que influenciam o processo de ensino e aprendizagem de matemática na educação de surdos, foram selecionados 24 artigos, como consta no Quadro 1, a partir do uso da metodologia de revisão Methodi Ordinatio.

Nesta seleção foram contemplados os artigos mais citados, como também os estudos recentes, como justificado por Pagani et al. (2017), com tais pesquisas tem-se um contexto provável em que novos avanços foram publicados sobre o tema e em consequência novas contribuições.

Quadro 1: Estudos com melhor classificação no portifólio

\begin{tabular}{|c|c|c|}
\hline $\mathbf{N}^{\mathbf{o}}$ & Autor & Título \\
\hline 1 & Holt (1994) & $\begin{array}{l}\text { Classroom attributes and achievement test scores for deaf and hard of } \\
\text { hearing students. }\end{array}$ \\
\hline 2 & $\begin{array}{l}\text { Zarfaty, Nunes, Bryant } \\
\text { (2004) }\end{array}$ & $\begin{array}{l}\text { The performance of young deaf children in spatial and temporal number } \\
\text { tasks. }\end{array}$ \\
\hline 3 & $\begin{array}{l}\text { Bull, Marschark, Blatto- } \\
\text { Vallee (2005) }\end{array}$ & SNARC hunting: Examining number representation in deaf students \\
\hline 4 & $\begin{array}{c}\text { Easterbrooks, } \\
\text { Stephenson (2006) }\end{array}$ & $\begin{array}{c}\text { An examination of twenty literacy, science, and mathematics practices } \\
\text { used to educate students who are deaf or hard of hearing }\end{array}$ \\
\hline 5 & Ansell, Pagliaro (2006) & $\begin{array}{l}\text { The relative difficulty of signed arithmetic story problems for primary } \\
\text { level deaf and hard-of-hearing students }\end{array}$ \\
\hline 6 & $\begin{array}{l}\text { Blatto-Vallee et al. } \\
(2007)\end{array}$ & $\begin{array}{l}\text { Visual-spatial representation in mathematical problem solving by deaf and } \\
\text { hearing students }\end{array}$ \\
\hline 7 & Kelly, Gaustad (2007) & $\begin{array}{l}\text { Deaf college students' mathematical skills relative to morphological } \\
\text { knowledge, reading level, and language proficiency }\end{array}$ \\
\hline 8 & Antia et al. (2009) & $\begin{array}{l}\text { Academic status and progress of deaf and hard-of-hearing students in } \\
\text { general education classrooms }\end{array}$ \\
\hline 9 & Kritzer (2009) & $\begin{array}{l}\text { Barely Started and Already Left Behind: A Descriptive Analysis of the } \\
\text { Mathematics Ability Demonstrated by Young Deaf Children }\end{array}$ \\
\hline 10 & Nunes et al. (2009) & Deaf children's informal knowledge of multiplicative reasoning \\
\hline 11 & Domahs et al. (2010) & $\begin{array}{l}\text { Embodied numerosity: Implicit hand-based representations influence } \\
\text { symbolic number processing across cultures }\end{array}$ \\
\hline
\end{tabular}




\section{Continua}

Quadro 1: Estudos com melhor classificação no portifólio (continuação)

\begin{tabular}{|c|c|c|}
\hline 12 & $\begin{array}{l}\text { Cannon, Fredrick, } \\
\text { Easterbrooks (2010) }\end{array}$ & $\begin{array}{c}\text { Vocabulary instruction through books read in American sign language for } \\
\text { English-language learners with hearing loss }\end{array}$ \\
\hline 13 & Spaepen et al. (2011) & Number without a language model \\
\hline 14 & Qi, Mitchell (2012) & $\begin{array}{l}\text { Large-Scale Academic Achievement Testing of Deaf and Hard-of-Hearing } \\
\text { Students: Past, Present, and Future }\end{array}$ \\
\hline 15 & $\begin{array}{c}\text { Garberoglio, Gobble, } \\
\text { Cawthon (2012) }\end{array}$ & A National Perspective on Teachers' Efficacy Beliefs in Deaf Education \\
\hline 16 & Pagliaro, Kritzer (2013) & $\begin{array}{l}\text { The Math Gap: A Description of the Mathematics Performance of } \\
\text { Preschool-aged Deaf/Hard-of-Hearing Children }\end{array}$ \\
\hline 17 & Marscharke et al. (2013) & Are deaf students visual learners? \\
\hline 18 & $\begin{array}{l}\text { Lange, Lane-Outlaw, } \\
\text { Lange y Sherwood } \\
(2013)\end{array}$ & $\begin{array}{l}\text { American sign language/english bilingual model: A longitudinal study of } \\
\text { academic growth }\end{array}$ \\
\hline 19 & Marschark et al. (2015) & $\begin{array}{c}\text { Predicting the Academic Achievement of Deaf and Hard-of-Hearing } \\
\text { Students From Individual, Household, Communication, and Educational } \\
\text { Factors }\end{array}$ \\
\hline 20 & $\begin{array}{l}\text { Hrastinski, Wilbur } \\
\text { (2016) }\end{array}$ & $\begin{array}{l}\text { Academic achievement of deaf and hard-of-hearing students in an } \\
\text { ASL/English bilingual program }\end{array}$ \\
\hline 21 & Jannah, Prahmana (2019) & $\begin{array}{l}\text { Learning fraction using the context of pipettes for seventh-grade deaf-mute } \\
\text { student }\end{array}$ \\
\hline 22 & Elsayed, Rakza (2020) & $\begin{array}{l}\text { The Relationship between Hyperactivity and Mathematics Learning } \\
\text { among a Child with Deep Deafness }\end{array}$ \\
\hline 23 & $\begin{array}{c}\text { Cuenca, Cervan, Cuberos } \\
\text { (2020) }\end{array}$ & Do Deaf Learners Reach the Necessary Linguistic Comprehension? \\
\hline 24 & $\begin{array}{l}\text { Madalena, Correa, } \\
\text { Spinillo (2020) }\end{array}$ & $\begin{array}{c}\text { Mathematical knowledge and language in deaf students: The relationship } \\
\text { between the recitation of a numerical sequence and Brazilian sign } \\
\text { language proficiency }\end{array}$ \\
\hline
\end{tabular}

Fonte: Elaboração dos autores, 2020

Na relação estabelecida em sala de aula, Soares e Sales (2018) destacam que o professor precisa ter sensibilidade para estreitar os laços de confiança, incentivo, motivação e reciprocidade com o aluno surdo. Em outras palavras, verifica-se que o cenário, em que o surdo está inserido, é circunscrito por diferentes fatores que podem influenciar em seu desenvolvimento.

$\mathrm{Na}$ educação de surdos, a falta de conhecimento da língua de sinais no ambiente familiar traz limitações para o acesso à informação (Sales; Soares, 2018). Nesse sentido as pesquisas demonstraram, a partir dos seus resultados que a fluência em língua de sinais (Hrastinski; Wilbur, 2016), o contato desde cedo com a língua (Madalena et al., 2020), a relação entre o nível de leitura e o conhecimento das palavras ( Kelly; Gaustad 2007) e a educação bilíngue (Lange et al., 2013) proporcionam melhores resultados no desempenho em sala de aula. 
A comparação entre o desempenho dos estudantes surdos e seus pares ouvintes aponta atraso em habilidades matemáticas (Bull et al., 2005), dificuldades nas operações básicas (Elsayed \& Rakza, 2020) e na interpretação de situações problemas (Blatto-Vallee et al., 2007; Kritzer, 2009; Pagliaro; Kritzer, 2013; Cuenca et al., 2020), fraco desempenho em raciocínio multiplicativo (Nunes et al., 2009).

De acordo com Blatto Vallee et al. (2007), em todos os níveis, o desempenho em matemática dos alunos ouvintes foi melhor do que os estudantes surdos. Diante dos testes padronizados, Qi e Mitchell (2012) afirmam que é preciso um olhar atento e um exame minucioso em relação aos resultados, pois leva-se em conta o que é abordado no currículo e de que forma, como também quais são as condições de ensino para os estudantes surdos.

O desempenho em matemática dos alunos surdos, segundo Holt (1994), que estudavam em escolas regulares, foi superior. Nas pesquisas de Antia et al. (2009) e Marschark et al. (2015), os autores constataram que os estudantes das escolas regulares apresentaram um bom aproveitamento, a relacioná-lo a uma série de fatores complexos envolvidos na aprendizagem, como a participação em sala de aula, os meio de comunicação e a participação dos pais, nas atividades e experiências no ambiente acadêmico.

A falta de convívio com colegas reduz o contato com a língua de sinais, como ensinam Soares e Sales (2018), pois reflete em um deslocamento social e interfere na aprendizagem e na compreensão do mundo que os rodeia. Como complementam Segadas et al. (2018), nem todos os surdos têm conhecimento, contatos com a língua de sinais e acessos à comunidade surda. Os estudos de Spaepen et al. (2011) mostraram que, mesmo diante de uma cultura numerada, aos indivíduos não receberem contribuições de uma língua convencional, afeta-se a compreensão de números exatos grandes.

A exploração dos recursos visuais aliada à comunicação em língua de sinais, ou seja à pedagogia visual, são caminhos para que a educação dos surdos contemple o processo de ensino e aprendizagem (Karnopp et al., 2019). Os alunos surdos apresentaram desempenho superior em tarefas com representação espaciais (Zarfaty et al., 2004), também experiências corporais, como a habilidade de contagem nos dedos, apresentou influência na representação numérica abstrata para surdos adultos (Domahs et al., 2010).

De acordo com Marscharke et al. (2013) são necessárias pesquisas mais profundas sobre como a habilidade visual-espacial afeta o aprendizado, para, a partir disto, obter 
direcionamentos a utilizar os pontos fortes desta situação. Os autores explicam que os estudantes surdos não apresentam tais habilidades superiores aos estudantes ouvintes, e complementam: “Os resultados encontrados sugerem que a preferência dos alunos surdos pela apresentação visual de informações não significa necessariamente que elas apoiam seu aprendizado mais do que os ouvintes" (Marschark et al., 2013, p. 161).

Para Ansell e Pagliaro (2006) os estudantes surdos e deficientes auditivos apresentam maneiras diferentes de responder às situações problemas em relação aos pares ouvintes. Os alunos surdos apresentaam dificuldades na decisão de quais operações deveriam usar na resolução do problema e não na história que compõe o problema.

A interação entre professores e alunos e o uso de recursos tecnológicos possibilitam a compreensão dos conteúdos do currículo, aliada às ferramentas visuais que potencializam a aprendizagem (Arroio et al., 2016). O uso de materiais concretos e os contextos aplicados que façam parte da realidade dos estudantes surdos, potencializam as experiências em sala de aula (Jannah \& Prahmana, 2019). As tecnologias também ampliam as possibilidades para o domínio de vocabulário, importante para a aprendizagem, como foi utilizado por Cannon et al. (2010) ao explorar DVD com histórias em língua de sinais para a área da Matemática.

$\mathrm{Na}$ formação inicial e continuada de professores diversas competências são discutidas, e cada vez mais isso se torna um desafio ao considerar os processos de inclusão dos estudantes. No contexto da educação de surdos, Easterbrooks e Stephenson (2006) mostraram que abordagem bilíngue, a qualificação do professor, a aprendizagem ativa com o uso de estratégias visuais e ferramentas que desafiem os estudantes, os recursos tecnológicos e as instruções a partir de situações problema, assim como o desenvolvimento do pensamento crítico, a ampliação do vocabulário e mediação são práticas de sucesso na sala de aula. As crenças coletivas de eficácia dos docentes na educação de surdos mostram resultados significativos para a importância das relações estabelecidas no ambiente escolar, como o clima, o poder de decisão e a colaboração entre os envolvidos (Garberoglio et al., 2012).

Diante do cenário descrito, verifica-se que a educação de surdos transita em um ambiente de múltiplas possibilidades e fatores que interferem diretamente no processo de ensino e aprendizagem de matemática em sala de aula. As pesquisas contribuem ao apontar as falhas e os possíveis caminhos para o futuro da educação, bem como as conquistas e aos exemplos que são mais explorados. 


\section{Considerações Finais}

Neste artigo, investigaram-se as abordagens das pesquisas na interface ensino de matemática e a educação de surdos, a partir de uma revisão sistemática da literatura para a seleção de construção do portifólio final de estudo. Nesse sentido foram levantadas duas questões de pesquisa: Quais temas são investigados e publicados sobre a interface educação de surdos e ensino de matemática? Quais fatores influenciam o processo de ensino e aprendizagem de matemática para estudantes surdos?

Este estudo de revisão sistemática da literatura apresentou o panorama dos estudos sobre o ensino de matemática na educação de estudantes surdos, de modo a contribuir para o campo de pesquisa, a sistematizar os principais autores e periódicos sobre o tema, assim como as discussões que permeiam o campo de estudo.

Para responder à primeira questão foram analisados 110 estudos sobre a educação de surdos e o ensino de matemática, a demonstrar que os temas mais abordados são o desempenho em matemática, o processo de ensino e aprendizagem de matemática na sala de aula, o uso dos recursos tecnológicos e suas diversas possibilidades que auxiliam a aprendizagem, como também a relação entre a fluência de língua de sinais e a matemática. Os estudos, em sua maioria, são de pesquisadores dos Estados Unidos, com destaque também para a abordagem com relação ao desempenho dos estudantes surdos e deficientes auditivos em testes padronizados.

Com relação à segunda questão, foram selecionados 24 estudos mais relevantes no portifólio e dentre os fatores que influenciam, os pontos convergentes são, o ensino e a aprendizagem de matemática em língua de sinais, com desenvolvimento de ferramentas visuais e a construção de um ambiente acessível para os alunos surdos, com respeito e valorização da cultura surda.

Diante desse contexto, compreende-se que mais pesquisas devem ser realizadas, a explorar os diferentes ambientes de aprendizagem e as condições dos espaços escolares, a usar a literatura já produzida como base para avanços na publicação científica e seus reflexos na sala de aula. Como indagações futuras a serem desenvolvidas, apresentam-se: Em quais condições as avaliações externas, a partir de testes padronizados, são aplicadas para estes estudantes? Como tornar esta avaliação acessível para os alunos surdos? A tradução e a interpretação para língua de sinais satisfazem as condições para a avaliação do desempenho escolar dos estudantes 
surdos? Estas são questões que necessitam ser discutidas e exploradas dentro do ambiente escolar, por toda a comunidade acadêmica.

\section{Agradecimentos}

À UTFPR pelo apoio no desenvolvimento da pesquisa.

O presente trabalho foi realizado com o apoio do Conselho Nacional de Desenvolvimento Científico e Tecnológico (CNPq), Brasil. Bolsista do CNPq, Brasil.

\section{Referências}

Adamo-Villani, N., \& Wilbur, R. (2010). Software for math and science education for the deaf. Disability and Rehabilitation Assistive Technology, 5(2), 115-124. doi: 10.3109/17 483100903387499

Adamo-Villani, N., \& Hayward, K. (2011). Signing Avatars. In: S. Hai-Jew. (Ed.), Virtual Immersive and 3D Learning Spaces: emerging technologies and trends. (pp. 249-267). New York, Estados Unidos: Information Science Reference.

Ansell, E., \& Pagliaro, C. M. (2006). The relative difficulty of signed arithmetic story problems for primary level deaf and hard-of-hearing students. Journal of Deaf Studies and Deaf Education, 11(2), 153-170. doi: 10.1093/deafed/enj030

Antia, S. D., Jones, P. B., Reed, S., \& Kreimeyer, K. H. (2009). Academic status and progress of deaf and hard-of-hearing students in general education classrooms. The Journal of Deaf Studies and Deaf Education, 14(3), 293-311. doi: 10.1093/deafed/enp009

Arroio, R. dos S., Pereira, A. L. M., Pinto, G. M. da F., \& Esquincalha, A. da C. (2016). Ensino de Matemática para o aluno surdo: revendo concepções e construindo paradigmas. Revista Paranaense de Educação Matemática, 5(9), 248-269.

Barbosa, H. H. (2013). Habilidades matemáticas iniciais em crianças surdas e ouvintes. Caderno Cedes, 33(91), 333-347. doi: 10.1590/S0101-32622013000300003

Barbosa, H. H. (2014). Conceitos matemáticos iniciais e linguagem: um estudo comparativo entre crianças surdas e ouvintes. Educação e Pesquisa, 40(1), 163-179. doi: 10.1590/S151797022014000100011

Blatto-Vallee, G., Kelly, R. R., Gaustad, M. G., Porter, J. \& Fonzi, J. (2007). Visual-spatial representation in mathematical problem solving by deaf and hearing students. Journal of Deaf Studies and Deaf Education, 12(4), 432-448. doi: /10.1093/deafed/enm022

Borba, M. C., Almeida, H. R. F. L., \& Gracias, T. A. de S. (2018). Pesquisa em ensino e sala de aula: diferentes vozes em uma investigação. Belo Horizonte, Brasil: Autêntica editora.

Bull, R., Marschark, M., \& Blatto-Vallee, G. (2005). SNARC hunting: Examining number representation in deaf students. Learning and Individual Differences, 15(3), 223-236. doi: 10.1016/j.lindif.2005.01.004 
Cannon, J. E., Fredrick, L. D., \& Easterbrooks, S. R. (2010). Vocabulary instruction through books read in American Sign Language for English-language learners with hearing loss. Communication Disorders Quarterly, 31(2), 98-112. doi: 10.1177/1525740109332832

Costa, W., C. L. da, \& Silveira, M. R. A. da. (2019). Aprendizagem das operações matemáticas fundamentais por alunos surdos usuários da Libras. Educação Matemática em Revista, 24(65), 128-142.

Cuenca, A. G., Cervan, R. L., \& Cuberos, M. P. (2020). Do Deaf Learners Reach the Necessary Linguistic Comprehension? International Journal of Disability, Development and Education, 67(1), 92-106. doi: 10.1080/1034912X.2019.1682527

Culbertson, L. (1974). CAI-Beneficial Teaching Tool at Texas School for the Deaf. American Annals of the Deaf, 119(1), 34-40.

D’Ambrosio, U. (2018). To think in a New Way in Mathematics Education. In: A. J. Riberio, L. Healy, R. E. de S. R. Borba, S. H. A. A. Fernandes (Eds.). Mathematics Education in Brasil, (pp 1-20), Springer.

Dessbesel, R. da S., Silva, S. de C. R. da, \& Shimazaki, E. M. (2018). O processo de ensino e aprendizagem de Matemática para alunos surdos: uma revisão sistemática. Ciência \& Educação (Bauru), 24(2), 481-500. doi: 10.1590/1516-731320180020014

Domahs, F., Moeller, K., Huber, S., Willmes, K., \& Nuerk, H. C. (2010). Embodied numerosity: implicit hand-based representations influence symbolic number processing across cultures. Cognition, 116(2), 251-266. doi: 10.1016/j.cognition.2010.05.007

Easterbrooks, S. R., \& Stephenson, B. (2006). An examination of twenty literacy, science, and mathematics practices used to educate students who are deaf or hard of hearing. American annals of the deaf, 151(4), 385-397.

Elsayed, S. A. \& Rakza, S. M. (2019). The Relationship between Hyperactivity and Mathematics Learning among a Child with Deep Deafness. International Electronic Journal of Mathematics Education, 15(1), em0562. doi: 10.29333/iejme/5951

Fernandes, S. H., \& Healy, L. (2013). Expressando generalizações em Libras: álgebra nas mãos de aprendizes surdos. Cadernos Cedes, 33(91), 349-368. doi: 10.1590/S010132622013000300004

Garberoglio, C. L., Gobble, M. E., \& Cawthon, S. W. (2012). A national perspective on teachers' efficacy beliefs in deaf education. Journal of deaf studies and deaf education, 17(3), 367-383. doi: 10.1093/deafed/ens014

Gravemeijer, K., Stephan, M., Julie, C., Lin, F.-L., \& Ohtani, M. (2017). What Mathematics Education May Prepare Students for the Society of the Future?. International Journal of Science and Mathematics Education 15, 105-123. doi: 10.1007/s10763-017-9814-6

Hrastinski, I., \& Wilbur, R. B. (2016). Academic achievement of deaf and hard-of-hearing students in an ASL/English bilingual program. Journal of Deaf Studies and Deaf Education, 21(2), 156-170. doi: 10.1093/deafed/env072 
Holcomb, T. K. (2011). Compartilhamento de informações: um valor cultural universal dos surdos. En: L. B. Karnopp, M. Klein, M. L. Lunardi-Lazzarin. Cultura na contemporaneidade: negociações, intercorrências e provocações. (pp. 139-149). Canoas: Ulbra.

Holt, J. A. (1994). Classroom attributes and achievement test scores for deaf and hard of hearing students. American Annals of the Deaf, 139(4), 430-437.

Jannah, A. F., \& Prahmana, R. C. I. (2019). Learning fraction using the context of pipettes for seventh-grade deaf-mute student. Journal for the Education of Gifted Young Scientists, 7(2), 299-321. doi: 10.17478/jegys.576234

Karnopp, L. B., Pokorski, J. de O., \& Zanini, J. V. (2019). Narrativas sobre a docência na Educação de Surdos. The Especialist, 40, (3), 1-14. doi: 10.23925/2318-7115.2019v40i3a11

Kelly, R. R., \& Gaustad, M. G. (2007). Deaf college students' mathematical skills relative to morphological knowledge, reading level, and language proficiency. Journal of deaf studies and deaf education, 12(1), 25-37. doi: doi.org/10.1093/deafed/en1012

Kritzer, K. L. (2009). Barely started and already left behind: A descriptive analysis of the mathematics ability demonstrated by young deaf children. Journal of Deaf Studies and Deaf Education, 14(4), 409-421. doi: 10.1093/deafed/enp015

Kritzer, K. L., \& Pagliaro, C. M. (2013). Matemática: um desafio internacional para estudantes surdos. Cadernos Cedes, 33(91), 431-439. doi: 10.1590/S0101-32622013000300008

Lange, C. M., Lane-Outlaw, S., Lange, W. E., \& Sherwood, D. L. (2013). American Sign Language/English bilingual model: A longitudinal study of academic growth. Journal of Deaf Studies and Deaf Education, 18(4), 532-544. doi: 10.1093/deafed/ent027

Madalena, S. P., Correa, J., \& Spinillo, A. G. (2020). Mathematical knowledge and language in deaf students: The relationship between the recitation of a numerical sequence and Brazilian Sign Language proficiency. Estudos de Psicologia, 37, e180175. doi: 10.1590/1982$0275202037 \mathrm{e} 180175$

Marschark, M., Morrison, C., Lukomski, J., Borgna,G., \& Convertino, C. (2013). Are deaf students visual learners? Learning and individual differences, 25, 156-162. doi: 10.1016/j.lindif.2013.02.006

Marschark, M., Shaver, D. M., Nagle, K. M., \& Newman, L. A. (2015). Predicting the academic achievement of deaf and hard-of-hearing students from individual, household, communication, and educational factors. Exceptional children, 81(3), 350-369. doi: $10.1177 / 0014402914563700$

Mendes, L. O. R., Bueno, A. J. A., Dessbesel, R. da S., \& Silva, S. de C. R. da. (2019). Gamificação no Processo de Ensino e Aprendizagem de Estudantes Surdos: uma revisão sistemática. RENOTE-Revista Novas Tecnologias na Educação, 17(3), 132-141. doi: 10.22456/1679-1916.99434

Moores, D. F. (2017). Research Methodology in Deaf Education early efforts. In: S. W. Cawthon, C. R. Garberoglio. Research in Deaf Education: contexts, challenges, and considerations. (pp.35-53). New York, Estados Unidos: Oxford University Press. 
Nunes, T., Bryant, P., Burman, D., Bell, D., Evans, D., \& Hallett, D. (2009). Deaf children's informal knowledge of multiplicative reasoning. Journal of deaf studies and deaf education, 14(2), 260-277. doi: 10.1093/deafed/enn040

Pagani, R. N., Kovaleski, J. L. \& Resende, L. M. (2015). Methodi Ordinatio: a proposed methodology to select and rank relevant scientific papers encompassing the impact factor, number of citation, and year of publication. Scientometrics, 105(3), 2109-2135. doi: 10.1007/s11192-015-1744-X

Pagani, R. N., Kovaleski, J. L., \& Resende, L. M. (2018). TICs na composição da Methodi Ordinatio: construção de portfólio bibliográfico sobre Modelos de Transferência de Tecnologia: Brapci 2.0. Ciência da Informação, 46(2), p.161-187. doi: 10.18225/ci.inf..v47i1.1886

Pagliaro, C. M., \& Kritzer, K. L. (2013). The math gap: A description of the mathematics performance of preschool-aged deaf/hard-of-hearing children. Journal of deaf studies and deaf education, 18(2), 139-160. doi: 10.1093/deafed/ens070

Peixoto, J. (2015). Gestos, sinais e esquemas de aprendizes surdos na multiplicação. Revista latinoamericana de investigación en matemática educativa, 18(3), 359-386. doi: 10.12802/relime.13.1833.

Qi, S., \& Mitchell, R. E. (2012). Large-scale academic achievement testing of deaf and hardof-hearing students: Past, present, and future. Journal of deaf studies and deaf education, 17(1), 1-18. doi: 10.1093/deafed/enr028

Ríos, N. M. B., López, D. F. G., \& Gallo, E. A. (2013). Representación de problemas matemáticos asociados al uso del algoritmo de signación en población sorda. Pensamiento Psicológico, 11(2), 39-52.

Ríos, N. M. B., Guimarães, L. S. P. \& Dorneles, B. V. (2018). Achievement in estimation of deaf students: a comparison between students from Brazil and Colombia. Educação, 43(4), 843-866.

Sacks, O. W. (2010). Vendo Vozes: uma viagem ao mundo dos surdos. Tradução Laura Teixeira Motta. São Paulo: Companhia das Letras.

Spaepen, E., Coppola, M., Spelke, E. S., Carey, S. E., \& Golsin-Meadow, S. (2011). Number without a language model. Proceedings of the National Academy of Sciences, 108(8), 31633168. doi: 10.1073/pnas.1015975108

Sales, L. M. (2009). Tecnologias digitais na educação matemática de surdos em uma escola pública regular: possibilidades e limites (dissertação de mestrado). Pontifícia Universidade Católica de Minas Gerais, Belo Horizonte, Brasil.

Sales, E. R. de., Penteado, M. G., \& Moura, A. Q. (2015). A Negociação de Sinais em Libras como Possibilidade de Ensino e de Aprendizagem de Geometria . Bolema, 29(53), 12681286. doi: 10.1590/1980-4415v29n53a23

Segadas, C., Bernardo, F. G., Moreira, C. dos S., Barbosa, P. M., Santos, R. C. dos., \& Garcez, W. R. (2018). Atividades de contagem com adaptações para alunos surdos e com deficiência visual. Rio de Janeiro: IM/UFRJ. 
Scheetz, N. A. (2012). Deaf Education in the 21st century: topics and trends. 1 ed. New Jersey: Pearson.

Soares, M. E., \& Sales, E. R. (2018). Das memórias às ideias: orientações sobre a visualidade na Educação Matemática para surdos. REPPE, 2(1), 61-90.

Zarfaty, Y., Nunes, T., \& Bryant, P. (2004). The performance of young deaf children in spatial and temporal number tasks. Journal of deaf studies and deaf education, 9(3), 315-326. doi: 10.1093/deafed/enh034 